\title{
Estigma y discriminación en los servicios de salud a las mujeres trabajadoras sexuales en América Latina y el Caribe
}

Stigma and discrimination in health services of female sex workers in Latin America and the Caribbean

\author{
Mario Pecheny \\ Profesor Titular de Sociología de la Salud. Universidad de Buenos Aires y CONICET, Buenos Aires (Argentina) \\ pecheny.mario@gmail.com
}

\section{CUERPOS, SEXUALIDADES YPODER}

MONOGRÁFICO COORDINADO POR JOSÉ MARÍA VALCUENDE. Universidad Pablo de Olavide (Sevilla).

\begin{abstract}
RES UMEN
El artículo tiene por objetivo describir las modalidades y magnitud del estigma y discriminación del que pudieran ser objeto las trabajadoras sexuales en relación con la atención de la salud, en América Latina y el Caribe. Sobre la base de 1006 encuestas a trabajadoras sexuales en 15 ciudades capitales de dicha región, realizadas en mayo-junio de 2013 por miembros de redes de trabajadoras sexuales, así como de 15 entrevistas colectivas semi-dirigidas a las trabajadoras sexuales que aplicaron las encuestas (en tanto informantes clave). El artículo describe y analiza los siguientes temas: características principales de hogar y familia de las trabajadoras sexuales; accesibilidad y demandas a los profesionales y servicios de salud; experiencias de estigma y discriminación en las consultas ginecológicas y de salud en general. Los resultados muestran que estas mujeres acuden en alta proporción a los servicios de salud, y que las experiencias son variables. Si bien se registran experiencias de estigma y discriminación, las trabajadoras sexuales manejan diversos recursos para evitar estas experiencias y conseguir una atención que consideran aceptable o incluso buena.
\end{abstract}

ABSTRAC T

This paper describes and analyses the situation of female sex workers in relation to stigma and discrimination in the health system in Latin America and the Caribbean. 1006 surveys (conducted in 15 capital cities) and 15 semi-directive interviews have been conducted in May-June 2013 to currently active sex workers. The results refer to: socio-demographic characteristics; accessibility and demands in health; experiences of stigma and discrimination in the context of the gynaecological consultation. These women attend health services in high proportions, and their experiences are variable. Experiences of stigma and discrimination are frequent, but these sex workers manage different resources to avoid stigma and gain good-quality attention and care.

PALABRAS CLAVE

trabajadoras sexuales |salud | estigma | América Latina | discriminación

KEYW O RDS

sexworkers | health | stigma | Latin America | discrimination

\section{Introducción}

El presente texto tiene por objetivo describir las modalidades y magnitud del estigma y discriminación del que pudieran ser objeto las trabajadoras sexuales en relación con la atención de la salud, en América Latina y el Caribe. Para ello, se realizaron 1006 encuestas a trabajadoras sexuales en las capitales (y sus áreas metropolitanas) de 15 países, en mayo-junio de 2013, así como 15 entrevistas colectivas semi-dirigidas a las trabajadoras sexuales que aplicaron las encuestas (consideradas en tanto informantes clave, ya que todas ellas son mujeres que están actualmente haciendo trabajo sexual) (1).

La iniciativa de la investigación surgió de las organizaciones de mujeres trabajadoras sexuales, a partir de sus experiencias personales como usuarias de los servicios de salud, que en muchos casos ha incluido situaciones de estigma y discriminación. En todos los países de la región pueden reconocerse problemas de accesibilidad y calidad de la atención en salud de las mujeres, ligados a deficiencias estructurales de los sistemas de salud, a las dificultades bastante generalizadas en relación con las mujeres de sectores populares y otras barreras socioeconómicas (Conill 2006, Pecheny y otros 2010). En esta investigación, nos centramos específicamente en las cuestiones de estigma y discriminación ligados al trabajo sexual. 
El estudio fue llevado a cabo en 2013, a iniciativa de la Red de Trabajadoras Sexuales de América Latina y el Caribe liderada por Elena Reynaga, bajo la coordinación del autor de este texto (REDTRASEX 2013). El objetivo principal del proyecto de investigación fue, junto al fortalecimiento de las capacidades de las organizaciones de trabajadoras sexuales de cada país de la región, conocer en qué medida y cómo el estigma y la discriminación asociados al trabajo sexual intervienen en la accesibilidad y la calidad de la atención en salud de las mujeres que se dedican a dicha actividad, en las áreas metropolitanas de las capitales de quince países: Argentina, Chile, Uruguay, Paraguay, Bolivia, Perú, Colombia, Ecuador, Panamá, Costa Rica, Honduras, Nicaragua, Guatemala, El Salvador y República Dominicana. Dado el número de encuestas realizadas en cada país (en promedio 70), el análisis se hará para el conjunto de la región, con alguna desagregación para el nivel sub regional. En el informe (REDTRASEX 2013) aparecen los datos desagregados para las subregiones y cada país, a título informativo.

Cabe hacer una aclaración previa acerca del contexto normativo. Todos los países que componen el estudio penalizan el proxenetismo -es decir, la explotación de la prostitución ajena; cuentan con legislaciones tendientes a combatir el delito de trata y tráfico de personas con fines de explotación sexual; y con la solitaria excepción de Uruguay, ninguno de los países reconoce legalmente el trabajo sexual. En todos ellos, es constante la persecución policial a las mujeres trabajadoras sexuales, a menudo basada en normativas nacionales o locales que directa o indirectamente criminalizan la oferta de sexo comercial (Morcillo y Justo von Lurzer 2012). La situación se ha visto agravada últimamente por la modalidad de implementación de las políticas contra la trata, que terminan por vulnerar los derechos de las trabajadoras sexuales (Bernstein 2010, Varela 2013). En más de la mitad de los países hay normativas de salud que afectan a las trabajadoras sexuales, relativas al control de $\mathrm{VIH}$ e infecciones de transmisión sexual. En Uruguay, Guatemala, Chile, Bolivia, Argentina (en algunas provincias), Panamá, Colombia (para establecimientos privados) y Honduras, controles ginecológicos y de infecciones obligatorios para las trabajadoras sexuales se llevan adelante en centros de salud específicos. Las mujeres deben concurrir a estos centros con determinada frecuencia y acreditar los resultados de sus exámenes médicos en carnets u otro tipo de identificaciones.

\section{Métodos}

La investigación que da pie al artículo se basa en una estrategia mixta, con un instrumento cuantitativo (encuesta aplicada por mujeres trabajadoras sexuales miembros de la RedTraSex a mujeres trabajadoras sexuales en actividad, con un pequeño componente cualitativo de asociación de palabras) y uno cualitativo (entrevistas realizadas por miembros del equipo de investigación a 44 trabajadoras sexuales consideradas como informantes clave). La encuesta ha permitido conocer la magnitud y variabilidad de experiencias de estigma y discriminación en relación con los servicios de salud, y las entrevistas ha permitido comprender mejor algunos resultados que pueden ser contradictorios. Por ejemplo, si los datos cuantitativos acerca de la última consulta muestran que las mujeres han ido en su inmensa mayoría a algún servicio de salud en el último año (lo que señalaría que las trabajadoras sexuales están en contacto con el sistema de salud) y que consideran en términos aceptables, o incluso muy buenos la calidad de la atención recibida, los datos cualitativos surgidos en las entrevistas permiten comprender que esto se explica por una gran diversidad de estrategias desplegadas por las mujeres a lo largo de su trayectoria como usuarias de los servicios: búsqueda de (o lucha por obtener) servicios y profesionales amigables (por lo cual la última consulta ha sido probablemente en aquel servicio que se ha revelado menos hostil o más amigable), manejo de la información acerca de la actividad que realizan, habilidad para conciliar horarios y espacios, etc.

Para el trabajo de campo y el análisis (REDTRASEX 2013), la muestra regional de América Latina y el Caribe se divide en tres sub-regiones: 1) Cono Sur (Argentina, Chile, Paraguay y Uruguay), 2) Andina (Bolivia, Colombia, Ecuador y Perú) y 3) América Central y Caribe (Costa Rica, El Salvador, Guatemala, Honduras, Nicaragua, Panamá y República Dominicana). La encuesta, no-probabilística, se aplicó en las áreas metropolitanas de las ciudades capitales de cada uno de estos países.

Se incluyó a mujeres trabajadoras sexuales en actividad al momento de la encuesta, de 18 años o más. Se excluyeron a varones y trans; a las mujeres de 17 años o menos; a las mujeres que no realizan actualmente trabajo sexual. Con el fin de contar con al menos 60 encuestas en cada país, número determinado por el tiempo disponible para hacer el campo y apuntando a garantizar un mínimo por país, se solicitó a cada encuestadora la realización de 22 encuestas, lo cual permitiría, llegado el caso, eliminar alguna encuesta 
muy incompleta, compensar si alguna compañera hacía un número menor, etc. Las trabajadoras sexuales que hicieron las encuestas participaron del diseño del instrumento de recolección de datos, en un encuentro que tuvo lugar en Buenos Aires. Sobre la base de un primer borrador con bloques de preguntas, se armó el cuestionario definitivo, se lo fue probando (mediante entrevistadoras del equipo de investigación primero, luego entre pares) para esclarecer cuestiones de contenido, forma y pases, y vocabulario, así como para incluir cuestiones que emergieron como significativas y excluir otras que fueron consideradas por las participantes como demasiado sensibles (por ejemplo, se eliminaron preguntas en materia de salud mental).

La muestra final quedó conformada por 1006 casos a nivel de América Latina y el Caribe en su conjunto. Todos los países superaron el mínimo de 60 encuestas previsto en el proyecto (se realizaron entre 63 y 75 encuestas por país). La recolección de datos fue realizada con precisión y corrección, lo cual favoreció la calidad de los datos y la consistencia de la encuesta. Las inconsistencias o datos faltantes pudieron subsanarse durante la etapa de supervisión, minimizando así la pérdida de información. Las encuestas fueron realizadas por mujeres actualmente realizando trabajo sexual, por lo cual tenían facilitado el acceso y la confianza por parte de las mujeres encuestadas. Las encuestas han sido en su mayoría efectuadas en el lugar y momento del trabajo, en los períodos de espera entre clientes. En algún caso, la encuesta fue interrumpida por la llegada de un cliente, y retomada luego. En todos los casos las encuestas fueron supervisadas por el equipo de investigación, con un muy bajo número de encuestas que debieron ser descartadas por incompletas. Dado el bajo número de encuestas por país, no se desagregan datos a ese nivel, sino que se analizan para el conjunto de la región con los porcentajes desagregados por subregión incluidos en cuadros.

Se realizaron, además, una serie de entrevistas semiestructuradas colectivas a informantes clave, representantes de las Organizaciones nacionales de trabajadoras sexuales, en relación a los objetivos generales del estudio, en mayo de 2013. En total fueron 15 entrevistas colectivas por país, a tres mujeres de cada uno de ellos con excepción de Panamá (de donde hubo dos entrevistadas). Las entrevistas, a partir de una guía de pautas, duraron entre 45 y 120 minutos en un único encuentro y fueron grabadas, y desgravadas, previo consentimiento de las entrevistadas. Todas ellas son residentes y nacidas en el respectivo país, y son trabajadoras sexuales en actividad actualmente. Además se hizo una discusión colectiva entre todas las informantes que fue grabada, desgrabada y analizada. En este artículo se consigna entre paréntesis como único dato el país de la entrevistada, puesto que especificar otras características podría atentar contra el anonimato de las respuestas (se trata de miembros de las organizaciones que públicamente han participado del estudio como encuestadoras, y por ende dar algún dato particular las identificaría; en el caso de las entrevistas, han hablado de sus propias experiencias como mujeres trabajadoras sexuales que se atienden en el sistema de salud, y de experiencias de sus compañeras).

Los resultados que se exponen en este informe se refieren, salvo aclaración expresa, a las mujeres residentes en el país o subregión, y no a la nacionalidad o lugar de nacimiento de las mujeres. Prácticamente en todos los países hay, además de las trabajadoras sexuales nacidas en el país donde se hizo la encuesta, un número variable de mujeres encuestadas que son extranjeras.

\section{Resultados}

En primer lugar, se exponen las características sociodemográficas de la muestra total de mujeres trabajadoras sexuales encuestadas; y en segundo lugar, los datos referidos a la atención en salud.

El promedio de edad general de las mujeres encuestadas es de 33 años. Las más jóvenes tienen 18 años de edad, límite inferior para ser incluidas en la muestra; la de mayor edad tiene 65 años. El rango de edad más frecuente es entre los 21 y 30 años, seguido por el rango de 31 y 40 años; y un 19,5\% tiene más de 40 años; estando los porcentajes más bajos en las más jóvenes entre 18 y 20 años $(6,7 \%)$, lógicamente ya que se trata de un rango de edad más exiguo. Casi la totalidad de las mujeres encuestadas se encuentran, pues, en edad reproductiva.

Varias de las encuestadas no nacieron en el país de la encuesta. El estatus migratorio tiene consecuencias respecto de la accesibilidad al control y atención de la salud. En el total de la muestra, el $88,2 \%$ de trabajadoras sexuales es no migrante, es decir, nacieron en el país donde trabajan y fueron encuestadas; y 
el $11,8 \%$ de trabajadoras sexuales proviene de otros países.

En cuanto al nivel de instrucción alcanzado, la mayoría de las mujeres trabajadoras sexuales encuestadas, casi la mitad, terminaron la educación básica (suma de "primaria completa" y "secundaria sin terminar"). Respecto del nivel de instrucción más bajo, llama la atención que casi un $20 \%$ no terminó la escuela primaria y que un $8 \%$ afirma nunca haber asistido a la escuela: esos son datos muy preocupantes para una región como la de América Latina y el Caribe, en la cual se presupone la obligatoriedad de la educación primaria y la accesibilidad a la educación como un derecho constitucional. Respecto del nivel de instrucción más alto, un $18 \%$ logró terminar el nivel secundario y casi un $10 \%$ ha iniciado ( $\mathrm{y}$, algunas, terminado) estudios de nivel superior.

El $45,2 \%$ de la muestra se declara soltera, y un $14,9 \%$ novia pero no conviviente. Las separadas/divorciadas representan el $7,2 \%$ y las viudas el $3,6 \%$. Convive con sus parejas menos de un tercio de la muestra: el $24,2 \%$ en uniones de hecho y solo el $5 \%$ casadas. El reducido porcentaje de casadas indica restricciones entre las trabajadoras sexuales para entrar en la legalidad civil y/o que la convivencia en matrimonio puede significar la interrupción total o parcial de la actividad como trabajadora sexual.

El desafío de sostener sus hogares y la crianza de hijos e hijas, e incluso nietos y nietas, impulsa a ingresar o permanecer en el trabajo sexual. Un gran porcentaje $(89 \%)$ de las trabajadoras sexuales encuestadas tiene hijos, y el $80 \%$ de ellas los tienen a cargo (para el $20 \%$ restante se trata de hijos adultos y/o a cargo de algún otro familiar). La media (promedio) es entre 2 y 3 hijos por cada mujer. En la muestra total, el número máximo de hijos que tiene una mujer encuestada es de 10 hijos. Un cuarto del conjunto de la muestra tiene, además, al menos un nieto o nieta; y el $12,2 \%$ del total de encuestadas convive con y/o tiene al menos un nieto a cargo.

"La primera vez que fui a neurología me hicieron caminar, no me preguntaron en qué trabajaba... vine yo y les dije 'soy trabajadora sexual' y vino la señorita y empezó con los sermones y le dije 'si usted fuera madre soltera, no estuviera donde está y tal vez tiene la capacidad de tener un trabajo en otro lado y si se sintiera así como me siento yo, ¿qué haría?, usted creo que haría hasta lo imposible, yo no soy capaz de dar lástima para que me den trabajo. Aunque sea con mi cuerpo yo mantengo a mis hijos" (Guatemala).

Una proporción mayor al $10 \%$ de las encuestadas viven solas. Casi dos tercios $(64 \%)$ del total de mujeres encuestadas vive con al menos un hijo o hija. Un tercio vive (también) con la pareja, y porcentajes que bordean el $20 \%$ pueden vivir (también) con la madre y/o hermanos.

El trabajo sexual constituye para este universo de mujeres la única o principal fuente de ingresos personales. Algunas mujeres encuestadas empezaron con el trabajo sexual hace muy poco tiempo y otras llevan varias décadas en la actividad. Del total de la muestra, el 3,5\% empezó con el trabajo sexual en los meses previos a la encuesta y el 7,2\% apenas un año antes. La mayoría lleva aproximadamente una década. En el otro extremo, una encuestada lleva 43 años en el trabajo sexual; el 3\% de la muestra lleva 30 años o más en la actividad; y el $11 \%$ de la muestra lleva 20 años o más (este porcentaje incluye a las que llevan 30 años o más).

La media de edad al inicio del trabajo sexual es 22,7 años; y la mediana 21 años. Del total de las encuestadas, el 17,3\% empezó con el trabajo sexual a los 17 años o menos (el 8,1\% con 15 años o menos, es decir en situaciones de ilegalidad y abuso). Estos datos no refieren a la situación actual, pero indican la exposición temprana a riesgos para la salud en la trayectoria de estas mujeres. Poco más del $2 \%$ comenzó con el trabajo sexual a los 40 años o más; la que se inició en la actividad a mayor edad fue a los 51 años.

Un poco más de la tercera parte de la muestra tiene actualmente al menos un trabajo o una actividad remunerada fuera del trabajo sexual; mientras que dos tercios no. Respecto de cuáles son los otros trabajos, los porcentajes son dispersos, resaltando un $11 \%$ que trabaja como vendedoras, y un $7 \%$ dedicado a las labores domésticas propias de una empleada del hogar. Los porcentajes son sobre el total de la muestra.

La gran mayoría (85\%) afirma ser ella la sostén principal del hogar; solamente un $6 \%$ declara que quien sostiene principalmente el hogar es el cónyuge, y el $5 \%$ la madre o padre (3,2\% y 1,5\% respectivamente).

Salvo en Uruguay, el trabajo sexual en la región no es una actividad legalmente reconocida como tal, por lo 
cual la cobertura en salud ligada al trabajo formal no suele ser accesible directamente para estas mujeres (indirectamente, podría ser a través del empleo del cónyuge o de algún familiar). En relación a la principal cobertura de salud, las categorías de respuesta son variadas, ya que muchas de las coberturas son mixtas o no tan definidas.

El sistema público de salud ofrecido por el Estado y que en muchos países es total o parcialmente gratuito cubre a casi $80 \%$ de las encuestadas. Un $10 \%$ tiene cobertura en la Obra Social, Seguro Social o sindical que corresponde a una cobertura ligada al trabajo formal (propio, o del cónyuge u otro familiar) o a otro tipo de aportes en materia de seguridad social laboral. Un $8 \%$ accede a médico particular pagando la atención de su propio bolsillo y $3 \%$ recurre a los planes privados de medicina pre-paga; a veces esto se da por elección pero en muchos casos es por desprotección social (no hay otra alternativa) y/o para evitarse situaciones de rechazo y discriminación en el sistema público. En algunos casos, cuando tienen los medios, las mujeres prefieren pagar el servicio y/o acudir a una clínica privada: para asegurarse una mejor atención y/o para evitar situaciones de hostilidad y estigma:

"Yo fui a un control ginecológico a hacerme un Papanicolaou, y no fue en algo público. Yo tengo seguro privado, es muy diferente porque cuando ya uno está pagando un servicio, no lo tratan igual" (República Dominicana).

“Es una clínica que cuesta, pero te tratan bien” (Panamá).

"No me atiendo con una mujer ginecóloga. Me siento bastante rechazada, o hasta ahora las veces que hemos tenido médicas ginecólogas, siento que es una cuestión de piel o una cuestión de género, el tema de que te tratan..., y más sabiendo que sos trabajadora sexual. Entonces, y capaz que es una idea mía, pero yo ya me predispongo a que me atienda mal. Y por eso elegí un médico ginecólogo (varón), y para eso voy a privado" (Argentina).

"En algún momento, tuve la necesidad de utilizar ese seguro, para las trabajadoras sexuales, y uno sabe que sí lo discriminan: lo vuelven a ver diferente, tiene que ir con más cuidado, le hablan grosero... Entonces yo, para no pasar por esas situaciones, ahora que puedo, entonces pago. Para cuando lo necesite, prefiero pagar" (Costa Rica).

Estos testimonios ilustran lo que se llama "anticipación de discriminación" (Pecheny 2003, Barbosa y Facchini 2008): cuando una persona supone o sabe que va a ser discriminada en algún contexto, se anticipa al rechazo y evita la situación. De ahí que si bien muchas mujeres señalan en la encuesta no haber sido efectivamente discriminadas en contextos de la atención en salud, esto se explica no tanto por ausencia en los servicios de discriminación hacia el trabajo sexual sino porque, o bien, evitan ir al servicio público, o bien, ocultan que se dedican al trabajo sexual. En ocasiones, esta información puede ser irrelevante (por ejemplo, para atenderse por una apendicitis) pero en otras ocasiones sí es relevante, como en la atención ginecológica o psicológica.

De Bolivia, donde la prevalencia del VIH entre las mujeres trabajadoras sexuales es muy baja, extraemos el siguiente fragmento:

"El trato en los centros de salud, en todos los departamentos, habrá uno o dos que no, pero siempre la doctora nos margina. Siempre nos pone como que nosotros somos el foco de infección. Y siempre, la población nos considera como que nosotros somos la población con más diagnóstico positivo en el tema de VIH" (Bolivia).

Los testimonios como éste muestran que las fuentes de estigma (Piscitelli 2006) se retroalimentan: estigma por pobreza, por género, por trabajo sexual y por $\mathrm{VIH}$. Conocimientos falsos y prejuicios contribuyen a ello, a menudo por parte de los propios profesionales de la salud.

Comencemos por un dato positivo que nos ha sorprendido al equipo de investigación. Ya sea porque hay regulaciones o prácticas que obligan a los controles de la salud, ya sea porque existe una preocupación por el cuidado y el autocuidado, resulta que casi la totalidad de las mujeres encuestadas $(98 \%)$ refieren haber acudido a un servicio de salud y/o a un médico/a en el último año.

En promedio, cada una de las trabajadoras sexuales encuestadas mencionó entre 4 y 5 motivos (no 
ginecológicos) por los cuales recurrió a un profesional o servicio de salud en el último año. La realización de algún análisis fue el motivo más mencionado (por el $76 \%$ de las trabajadoras sexuales encuestadas), seguido por controles voluntarios de salud que fueron mencionados por 7 de cada 10 encuestadas. Casi 4 de cada 10 de las trabajadoras sexuales encuestadas consultó en el último año por cuestiones relacionadas con el VIH o sida. Asimismo, el 31\% realizó consultas sobre infecciones de transmisión sexual. Las cuestiones respiratorias (resfríos, gripe, neumonía, etc.) fueron motivo de consulta para casi el $40 \%$ de las encuestadas. Un tercio de ellas realizó alguna consulta odontológica en el último año.

El alcance de la obligatoriedad de la realización de controles de salud entre las trabajadoras sexuales se ve reflejado en que el $32 \%$ de las encuestadas dijo que consultó con un profesional o servicio de salud en el último año "porque estaba obligada a controlarse por el carnet sanitario u otra disposición legal" y una similar proporción dijo que lo hizo "porque la obligaron en su trabajo a hacerse un control". Estos porcentajes son más altos en los países con reglamentaciones que así lo exigen.

Casi una de cada diez mujeres encuestadas acudió solidariamente a servicios de salud el último año para donar sangre.

Resulta preocupante la proporción cercana al 18\% de trabajadoras sexuales encuestadas que debió recurrir al médico o servicio de salud en el último año por haber sido víctima de golpes o violencia. Esto es aún más alarmante si tenemos en cuenta que, frecuentemente, las mujeres que son víctimas de violencia (por parte de la policía, de clientes, de sus parejas, de otros hombres, de otras trabajadoras sexuales) no acuden a los servicios en busca de asistencia para evitar situaciones de re-victimización y estigma, e incluso puede presuponerse que tampoco lo cuentan fácilmente en nuestra encuesta, lo cual indicaría que este $18 \%$ es solo la "punta del iceberg" de un problema de mayor envergadura.

"La última vez que fui a un servicio de salud, fue cuando tuve un accidente que me cortaron la pierna.

- ¿Cómo fue ese corte de pierna?

Un puntazo con un cuchillo.

- ¿Quién te atacó?

Una compañera, por celos. Por celos me atacó.

-¿Y cómo fue? Cuéntame, ¿qué paso? ¿Fue como una especie de ataque? Te cortó la pierna, y ¿tú qué hiciste?

No pues. Me llevaron para el hospital. Yo estaba tomada.

- Ya. Estabas en el momento del trabajo.

En el momento del trabajo, sí. Estaba tomada, ella llegó, me metió el puntazo y se fue. Ella se fue y me llevaron. Y me atendieron pero fueron muy ordinarios.

- ¿A ver, qué pasó cuando te atendieron? ¿Ellos sabían cuando tú llegaste con tu herida, ellos sabían que tú eras trabajadora sexual? ¿Cómo fue? ¿Cómo te trataron?

Fueron muy ordinarios, me decían 'cállese, déjese de ser tan cobarde, no grite'. Yo les decía, ‘jes que me duele! Me duele. Cómo no, si me duele'. No me gustó esas palabras que me dijeron.

- ¿Qué te dijeron?

‘Cállate!, deje de gritar'. No es que... yo no gritaba porque quería. Sino que yo sentía dolor. Yo le decía. Y pues, claro, por una parte tampoco tuvieron tanta culpa porque a mí no me cogió la anestesia por el trago, ¿me entiende?

- ¿Tenías mucho trago encima?

Sí, claro, estaba alcoholizada

- ¿Te decían algo por eso? Por esa razón que...

Es que me cogían muy brusco. O sea, le cogían la pierna duro a uno y eso me decían pues. Así, solamente me jalaban duro y mi compañera, la que me llevó, no le gustó, y dijo: ‘jay no, cómo la cogían de brusco a usted! No me gustó'. Y no pues, rechazos, sí.

- Volviendo un poco a tu accidente, ¿los médicos ahí, sabían a lo que tú te dedicabas? 
Sí, porque yo tenía historial. Yo ya, como a medida que uno entra, de una hacen que baje la historia de uno.

- ¿Y ellos comentaban algo con respecto a eso? ¿Por el tema de tu corte?

La verdad, no sé. Porque yo estaba ya después, en un estado... desmayada del dolor. La verdad no sé.

- ¿Y después qué te hicieron?

No. Solamente me cogieron, me limpiaron ahí. Me cogieron cuatro puntos por fuera, cuatro puntos por dentro y me mandaron para la casa" (Colombia).

El estigma del trabajo sexual atenta en muchos casos contra la accesibilidad y la calidad de la atención, mediante la culpabilización de la víctima:

"Donde yo me parqueo, como se dice allá, hay varias que han tenido heridas de clientes. A las guardias no pueden acudir, porque les dicen 'tú te la buscaste', y hasta la llevan presa aunque estés sangrando. Pero si van al médico normal la atienden.

- ¿Y les preguntan qué les pasó?, ¿les cuentan?

No les cuentan. Las atienden normal como a otro cualquiera" (Panamá).

No sólo las disposiciones legales obligan a las trabajadoras sexuales a realizarse controles de salud, sino también sus empleadores o locales privados donde trabajan. Este es el caso para una proporción importante de las encuestadas en Ecuador, Uruguay, Perú, Colombia y Bolivia.

Respecto de la salud ginecológica/reproductiva, un $89 \%$ de las trabajadoras sexuales encuestadas ha consultado al menos una vez en el último año. Las encuestadas mencionaron, en promedio, dos motivos cada una. El motivo de consulta más habitual es la realización de controles ginecológicos (por ejemplo, PAP y control de mamas), mencionado por el $87 \%$ de las trabajadoras sexuales encuestadas. La búsqueda de preservativos como motivo de consulta fue mencionada por poco más de la mitad de las encuestadas (varias aclararon, además, que suelen retirar preservativos en los controles).

Solo cuatro de cada diez ha solicitado atención en el último año en relación con métodos anticonceptivos. Una proporción menor, pero considerable, ha consultado por embarazo o parto en el último año (15\%), mientras poco más del $8 \%$ dijo haber consultado por complicaciones, pérdida o interrupción del embarazo.

Si bien la realización de controles ginecológicos frecuentes parece estar extendida entre las trabajadoras sexuales encuestadas, también se debe tener en cuenta la calidad y calidez de la atención que reciben. Algunos de los testimonios de las entrevistas dan cuenta de situaciones de evidente vulneración de derechos:

"Asistí a un centro de salud para hacerme un Papanicolaou hace como cinco meses, allí me detectaron un problema de ITS, para profundizar ese problema me mandaron a un hospital público de Managua, la capital. Fui, hice todos los papeles y entonces me metieron en un cuarto, cuando entré sólo estaba una doctora, que me iba hacer una colonoscopía y después entraron sin avisar tres doctoras más. Y yo estaba sin ropa y no me dieron nada para taparme, cuando entraron las tres yo me pues muy tensa, pero no dije nada, entonces la doctora que me estaba viendo llamó a las otras doctoras y les dijo que vieran lo que me estaba haciendo, lo que me estaba pasando. Cuando ella tomó la muestra, a mi me dolió demasiado, no podía hablar, yo sólo le dije por señal que no que no quería que me vieran las otras. Cuando terminó yo le dije porqué lo había hecho, que eso no estaba bien, que de ser así, ella debió avisarme. Que debió se discreta, que era ella la que iba a estudiar mi caso no las otras y que yo decidía porque yo era la paciente y yo decidí si aceptaba o no a otras médicas en mis exámenes. Ella se molestó porque yo le reclamé. Para entregarme el resultado ella no me dijo qué día llegar sino que le dijo a su asistente que me diera el resultado, no me pareció porque los servicios de salud no deberían ser así. Me sentí con mucha pena, me dio mucha vergüenza y a la vez sentí que no respetaron mis derechos A ninguna persona deberían hacer eso porque somos personas y deben respetar nuestros derechos" (Nicaragua).

“- ¿Alguna consulta, que haya sido particularmente significativa para ti?

Sí, pero no para conmigo. Este año pasado me llamó la atención el caso de una compañera que fue violada y entró dentro del marco de aborto no punible, y ver el tema de la excelente respuesta de los 
médicos, que quizás ya estaban sensibilizados.

- ¿Eso fue en un hospital público?

Fue en un CAPS (centro de atención primaria), un centro de salud público. Los profesionales ya estaban sensibilizados, ver lo que generó esa circunstancia, el trabajo interdisciplinario, el articular. El saber que en hospital nos rechazaban, nos rechazaban, nos rechazaban, o por los jefes de servicio, o por el tema del protocolo o esto y esto y esto. Daban vueltas. Y ver las gestiones que hacían los del centro de salud. El desafío de la lucha. $Y$ de defender el tema de que podes ser trabajadora y ser violada. Eso es algo que marcó, porque para nosotras fue un re logro como equipo de trabajadoras con un equipo de médicos que realmente se puso la camiseta. Fue la primera vez que vimos que realmente defendían el derecho de la trabajadora a no ser discriminada, a ser una ciudadana más. Porque la jefa del servicio del hospital donde tenían que hacer, que practicar, el aborto decía que si es prostituta no es una violación" (Argentina).

"En vez de que entre una por una, al control, que es donde nos revisan, entran como animales. De cinco en cinco, de seis en seis (...). Y hay una doctora en especial, que es la que agarra un papel; 'iponte bajo las nalgas! ¡Sácate el pantalón! ¡Que tiene que ser rápido! ¡Pero, ¿por qué?! ¿Ves? ¡Ya estás enferma! ¡Cochina!', y todo lo demás. Asi” (Bolivia).

La salud integral incluye la salud mental, a menudo relegada a un segundo plano, o limitada arbitrariamente a los malestares que pudiera ocasionar a la mujer dedicarse al trabajo sexual. La tercera parte de las trabajadoras sexuales encuestadas en el total de la región de América Latina y Caribe manifestó haber necesitado ayuda psicológica en el último año. Entre quienes sintieron esa necesidad $(n=338)$, solo la mitad recurrió efectivamente a un profesional o servicio de asistencia psicológica en el último año.

Esto refleja la todavía pendiente cuestión de incorporar la salud mental y el bienestar espiritual a la atención integral en salud, tanto desde la perspectiva de las usuarias como de las/os profesionales de la salud.

El no reconocimiento de la salud mental forma parte de una perspectiva que en general falla en reconocer la integralidad de la salud de las personas. Este fragmento de entrevista a trabajadoras sexuales lo grafica claramente:

"Que se dediquen a cuerpo entero. (...) Más que todo es vagina. Pero debería ser general.

- Claro, general.

Yo pienso y siempre le he dicho al jefe, médico jefe, de ese centro de salud para donde derivo, que la trabajadora sexual debería pasar cada mes con la psicóloga. ¿Por qué? Porque el trabajo sexual te da dinero, pero te enferma. Y te enferma de acá (señala la cabeza). ¿Sabes por qué? Porque hoy día por ejemplo voy a chambear, y no tengo ni un cliente, y estoy que (hace un ruido) $\mathrm{mmm}$...

Te desesperas.

Todo eso te va enfermando.

Te va enfermando. $Y$ peor, si tienes problemas jimagínate! $Y$ no tienes para pagar. Entonces yo creo que una consulta mensual con la psicóloga te ayudaría bastante, es más, te ayudaría a entender que tienes que cuidar tu cuerpo, que es tu herramienta de trabajo. Pienso que debería ser eso también, salud mental, vernos todo, todo lo relacionado con nuestro cuerpo, que eso es importante.

Porque no solo es, no somos vagina. Es pulmones, hígado, tantas cosas que tenemos, ¿no? (...)

Solamente...

De abajo.

La vagina...

La vagina nada más" (Perú).

Cuando tienen un problema de salud, y pesar de las dificultades mencionadas, el $75 \%$ de las encuestadas dijo mayoritariamente buscar un servicio de salud o ayuda profesional, y/o acudir a una farmacia (58\%). Asimismo, según la situación, por fuera del sistema de salud, antes de la ayuda profesional o en su reemplazo, más de la mitad de las trabajadoras sexuales prefiere arreglárselas sola y/o tomar medicamentos por su cuenta. Tres de cada diez pide ayuda a un familiar o esperan que se pase el problema y aproximadamente la cuarta parte recurre a otras trabajadoras sexuales, amigas $u$ organizaciones sociales. Un $8 \%$ suele pedir ayuda o preguntarle a algún cliente (se trata de una pregunta de respuesta múltiple). 
"Es una lata ir al médico. Es una lata. En realidad en Chile para ir al médico, para un turno tienes que levantarte como a las cuatro de la mañana para sacar una hora, un número en el consultorio. Cuando llegas hay una cola como de diez metros que da la vuelta a la manzana y ya no te quedan ganas de ir al médico. Si estás enfermo preferís automedicarte, que es lo que hago yo, o sencillamente no ir al médico" (Chile).

Este testimonio sobre el tema horarios da cuenta de una situación que no es exclusiva de las trabajadoras sexuales. En lo que sigue profundizamos aspectos específicos de la situación de estas últimas.

En el estudio se indagó sobre las características, calidad de la atención y satisfacción en relación con la última consulta sobre salud no-ginecológica y sobre salud reproductiva o ginecológica. Preguntar por la última consulta tienen la ventaja de que es más fácilmente recordada que otras realizadas en el pasado; y hay que aclarar que se trata pues, en muchos casos, de consultas realizadas con profesionales y/o servicios encontrados luego de peregrinajes más o menos largos escapando a quienes estigmatizan y procurando hallar entornos amigables. De ahí que los resultados sobre grado de satisfacción de las usuarias (bastante alto, como veremos) deban comprenderse en las trayectorias de mayor duración. En este texto, nos concentramos en la consulta ginecológica: el tema de la sexualidad y de la (no-)reproducción es allí central e indisociable de la actividad que llevan a cabo las mujeres consultantes.

Tres cuartos de la muestra de las trabajadoras sexuales encuestadas en toda la región realizaron la última consulta ginecológica en el transcurso del último año: $30 \%$ en el mes anterior a la encuesta y $46 \%$ hacía más de un mes pero menos de un año.

En cuanto al lugar de dicha consulta, un $73 \%$ acudió a un hospital público, siendo la opción mayoritaria como en el caso de la última consulta sobre salud general no-ginecológica. Una proporción cercana al 13\% consultó por última vez sobre salud reproductiva en el ámbito privado (8\% en clínica y $5 \%$ en un consultorio particular). La proporción de quienes realizaron la última consulta sobre salud reproductiva en un servicio o consultorio específico para trabajadoras sexuales es mayor que en el caso de la última consulta sobre salud general $(11 \%$ versus $4 \%)$. Los puestos de atención primaria ocupan un lugar minoritario, tal como en la última consulta sobre otras cuestiones de salud.

Algunas entrevistadas mencionan lugares específicos para trabajadoras sexuales. Estos varían mucho según los países, ciudades y servicios, desde aquellos que ofrecen atención amigable a aquellos que son francamente hostiles:

"Voy sola con el carnet de salud. Siempre me trataron bien. Siempre dije que era trabajadora sexual, yo estoy orgullosa de mi trabajo. Y yo me atiendo con la Dra. B., mi control de diabetes que me lo hicieron hace tres meses. Pero no tengo nada que decir, porque es de 'che', y 'vos', porque ya hace tantos años que me atiende la misma doctora... Y en profilaxis, en la clínica de ITS, es lo mismo, pero también, las doctoras ya hace veinte años que están. Nos conocen de toda la vida" (Uruguay).

“- ¿Cómo te sentiste o pasó algo importante en la última visita al ginecólogo o ginecóloga?

No, nada importante. Una cosa normal, como toda mujer que va. Además es un hospital donde vamos, ya nos atienden siempre los mismos y ya saben que somos trabajadoras sexuales, no tenemos que decir nada.

- Ah, ya saben... ¿Y eso cambió la atención o no?

No, para nada. Al contrario, nos tienen mucho respeto" (Argentina).

"Nosotras, como trabajadoras sexuales, hay un centro que se supone que es el que nos asignaron, pero se paga por todo y se aprovechan mucho de las compañeras" (República Dominicana).

La amigabilidad de los servicios y la mejor accesibilidad a los mismos se explican a veces por el trabajo de las redes y organizaciones de trabajadoras sexuales:

"En nuestro caso, nosotras tenemos nuestro centro amigable un día a la semana para las trabajadoras sexuales. En el Hospital A tenemos un día de ginecóloga, conseguimos un día de infectología. Entonces, eso se acompaña. Lo único que hay que hacer es el acompañamiento a las compañeras, porque solas no te van, entonces yo les tengo que estar arriba, ir a buscarlas a la puerta del hotel o golpearles la puerta. Hacerles acompañamiento. En este momento, eso se está haciendo mucho, el 
acompañamiento a las compañeras, porque no te van solas" (Argentina).

"Antes, a mí me costaba mucho ir a las consultas porque le hacían tantas preguntas y una tenía que decir que era trabajadora sexual y que lo vieran a una con ese rechazo, en ocasiones los doctores se ponían hasta doble guantes para atendernos" (Nicaragua).

"Si se va por sí sola es muy difícil. Las compañeras pasan momento difícil. No dicen que son trabajadoras sexuales. Si llega a decir que es una trabajadora sexual la discriminan automáticamente" (Paraguay).

Los controles (PAP, examen de mamas, etc.) son el principal motivo de la última consulta sobre salud reproductiva, mencionado por el $60 \%$ de las trabajadoras encuestadas en toda la región. El segundo motivo de la última consulta fueron las cuestiones relacionadas con el embarazo y el parto, por el 11 por cuento de las encuestadas.

Casi todas las trabajadoras sexuales encuestadas (más del 95\%) consideran que en la última consulta sobre salud reproductiva los profesionales las escucharon con atención y las trataron con respeto. Alrededor del $60 \%$ sintió que sus preguntas o dudas fueron tomadas en consideración por el profesional (ya sea haciendo lugar a las mismas o respondiéndolas), y poco más de la mitad refirió haber recibido apoyo emocional.

\begin{tabular}{|l|r|r|r|c|}
\hline \multicolumn{6}{|l|}{$\begin{array}{l}\text { Distribución en valores porcentuales de las mujeres trabajadoras sexuales encuestadas } \\
\text { por valoración positiva de dimensiones de calidad de atención en última consulta sobre } \\
\text { salud reproductiva, según subregión.* América Latina y Caribe, } 2013\end{array}$} \\
\hline Subregión & Cono Sur & Andina & $\begin{array}{c}\text { América } \\
\text { Central y } \\
\text { Caribe }\end{array}$ & $\begin{array}{c}\text { América } \\
\text { Latina y } \\
\text { el } \\
\text { Caribe }\end{array}$ \\
\hline La escucharon con atención & 95,1 & 94,0 & 97,1 & 95,8 \\
\hline La trataron con respeto & 98,8 & 95,7 & 93,3 & 95,4 \\
\hline $\begin{array}{l}\text { Le brindaron o le ofrecieron apoyo } \\
\text { emocional }\end{array}$ & 48,4 & 48,3 & 60,1 & 53,8 \\
\hline $\begin{array}{l}\text { Le preguntaron si tenia alguna duda o } \\
\text { pregunta }\end{array}$ & 69,3 & 56,4 & 47,0 & 55,5 \\
\hline Le respondieron sus dudas o preguntas & 74,2 & 58,5 & 52,5 & 60,0 \\
\hline
\end{tabular}

Fuente: Encuesta REDTRASEX 2013 (REDTRASEX 2013)

El alto grado de satisfacción con la última consulta se explica, al cruzarse con los testimonios recogidos en las entrevistas, por ser el resultado de largos recorridos en busca de servicios o profesionales amigables, recorridos por servicios y profesionales que pueden ser francamente hostiles, y en relación con los cuales se evalúa la última consulta.

"Yo hace muchos años, de unos 17 años o más, fui con un doctor. Ese día me hizo un Papanicolaou y esa vez me dijo que me quitara la blusa porque tenía que revisarme los pechos, yo me la quité porque como él es el que sabe, y me empezó a tocar los pechos y me dijo que me tocara la punta de los pechos porque esa es una revisión completa, yo me sentí incomoda pero lo hice y me decía 'no, así y me empezó a tocar los pechos. Yo me dejé pero sentí vergüenza porque uno está desnudo totalmente. Luego me dio mi receta. Yo me sentí acosada" (Guatemala).

Los relatos de las entrevistas cualifican mejor las situaciones. En algunos casos, por ejemplo, el hecho de que la paciente sea una trabajadora sexual parece habilitar al médico a no respetar demandas de privacidad o la integridad personal y corporal:

"Fui a hacerme un ginecológico de profilaxis, como le decimos en Ecuador, estaban los chicos practicantes, entonces yo le dije al doctor, entré y le dije, 'doctor no quiero que ellos me vean porque soy un poquito tímida y no me gusta que me vean'. Entonces me dijo: 'cuando trabajas no te acuestes con tíos porque es muy serio'. Nos quedamos viendo y discutimos" (Ecuador). 
"Y antes, había un doctor que no es que era grosero, sino que como sabía que eran trabajadoras sexuales les decía cosas como 'Súbase a la cama del placer'. Así. O sea, yo pienso que eso es un irrespeto. Así le decía a la camita. Eso no me lo dijo a mí, sino a una compañera, y ella le dijo: 'A mí me respeta'. Yentonces fue abajo y ella puso la denuncia" (Costa Rica).

Los estudios sobre satisfacción de usuarias y usuarios de servicios de salud suelen dar altos porcentajes de satisfacción (Petracci 2004), dados diversos factores: bajas expectativas previas, agradecimiento ante la resolución de preocupaciones por la salud, etc. Como otras poblaciones acostumbradas al maltrato (Barbosa y Facchini 2008, Pecheny 2010), también las trabajadoras sexuales suelen recorrer servicios y profesionales hasta hallar aquellos que sean menos hostiles o francamente amigables (de Paiva 2013): las mujeres se van pasando de boca en boca estas informaciones, recurso esencial para habilitar siquiera el acceso a la atención en salud.

La calidad de la atención en salud requiere de la comunicación, confianza, buena información y garantía de confidencialidad. Para la salud sexual y reproductiva de estas mujeres, que se hable o no del trabajo sexual de la consultante es clave para evaluar las condiciones de acceso y la calidad de la atención, y depende de esos factores.

Casi el $60 \%$ del total de las trabajadoras sexuales encuestadas consideró que era importante hablar sobre su actividad como trabajadoras sexuales en la última consulta sobre salud reproductiva. Esta proporción prácticamente duplica a la referida en el caso de la última consulta sobre salud general.

Pero más de la mitad de las mujeres trabajadoras encuestadas no habló del tema del trabajo sexual en la última consulta sobre salud reproductiva $(n=518)$. Solo el $44 \%$ sí lo hizo $(n=421)$. Como es de esperar, quienes más hablaron del tema son aquellas que consideraban importante hacerlo. Sin embargo, aun dentro de este grupo $(n=557)$, casi el $30 \%(n=154)$ no habló del tema del trabajo sexual en la última consulta sobre salud reproductiva.

Entre quienes no hablaron del tema del trabajo sexual en la última consulta sobre salud reproductiva $(n=518)$, el sentir vergüenza fue la razón más esgrimida, mencionada por el $44 \%$ (proporción similar e incluso levemente mayor que en la consulta sobre salud general). Además, más de un tercio dijo no haber hablado del tema por miedo al rechazo, así como por miedo a que se enteren otras personas. Una proporción cercana a un tercio dijo que no hubo oportunidad de hablar en la consulta y otro tanto que no lo hizo porque era algo ya sabido o conversado anteriormente.

Entre quienes sí hablaron del trabajo sexual en la última consulta sobre salud reproductiva ( $\mathrm{n}=421$ ), el $57 \%$ dijo haberse sentido aceptada al hacerlo, el $5 \%$ dijo haberse sentido rechazada y más de un tercio no tuvo ninguna sensación particular al respecto.

El tema del trabajo sexual no se incluye en la consulta de salud reproductiva, por ejemplo, en situación de embarazo, en una suerte de pacto de silencio tanto por parte de los profesionales de la salud (que no consideran la eventualidad del trabajo sexual) como por parte de las mujeres.

"Esos temas por lo regular no se tocan. No se tocan. Nadie te va a hablar de si tú eres trabajadora sexual o si estás en la... En lo que sea... Esos teman no se tocan porque no te lo van a contestar. Aunque sea cierto no te lo contestan" (Panamá).

"Los profesionales de salud empiezan con un montón de prejuicios, y cualquier cosa que tienes, algo en la boca, algo en la vagina, ya lo catalogan, que por ser trabajadora sexual lo tienes por eso. Si no es necesario decirlo, no lo decimos..." (República Dominicana).

¿Qué sucede en la trayectoria de estas mujeres como usuarias del sistema de salud? Se les consultó a las trabajadoras sexuales encuestadas sobre diferentes situaciones de estigma y discriminación en los servicios de salud, ligadas a su actividad. Los resultados se muestran en el cuadro siguiente: 


\begin{tabular}{|c|c|c|c|c|}
\hline \multicolumn{5}{|c|}{$\begin{array}{l}\text { Distribución en valores porcentuales de las mujeres trabajadoras sexuales } \\
\text { encuestadas por experiencias de estigma y discriminación en servicios de salud, } \\
\text { según subregión. Respuesta múltiple*. América Latina y Caribe, } 2013\end{array}$} \\
\hline Le pasó alguna vez que... & $\begin{array}{l}\text { Cono } \\
\text { Sur }\end{array}$ & Andina & $\begin{array}{l}\text { América } \\
\text { Central } \\
\text { y Caribe }\end{array}$ & $\begin{array}{l}\text { América } \\
\text { Latina y } \\
\text { el } \\
\text { Caribe }\end{array}$ \\
\hline $\begin{array}{l}\text { Se atendió lejos de donde vive para que en } \\
\text { su barrio o su casa, no supieran que es } \\
\text { trabajadora sexual }\end{array}$ & 46,7 & 68,1 & 58,9 & 58,1 \\
\hline $\begin{array}{l}\text { Se atendió lejos de donde realiza su } \\
\text { actividad como trabajadora sexual para } \\
\text { evitar que lo sepan en el servicio }\end{array}$ & 37,9 & 52,4 & 41,9 & 43,8 \\
\hline $\begin{array}{l}\text { La obligaron a hacerse un análisis porque } \\
\text { es trabajadora sexual }\end{array}$ & 20,0 & 60,1 & 33,3 & 37,3 \\
\hline $\begin{array}{l}\text { Tuvo dificultades para ir al hospital o } \\
\text { servicio de salud porque los horarios se } \\
\text { superponían con el trabajo }\end{array}$ & 45,4 & 35,1 & 31,8 & 36,5 \\
\hline $\begin{array}{l}\text { Le recomendaron en una consulta médica } \\
\text { que abandonara el trabajo sexual }\end{array}$ & 26,3 & 34,3 & 39,1 & 34,2 \\
\hline $\begin{array}{l}\text { No quiso ir al hospital o servicio de salud } \\
\text { para no tener que dar explicaciones sobre } \\
\text { su actividad }\end{array}$ & 26,7 & 33,1 & 37,0 & 33,0 \\
\hline $\begin{array}{l}\text { Sintió hostilidad por parte del personal } \\
\text { administrativo del servicio de salud, por } \\
\text { ser trabajadora sexual }\end{array}$ & 17,9 & 32,7 & 29,4 & 27,2 \\
\hline $\begin{array}{l}\text { Tuvo que cambiar de hospital o servicio de } \\
\text { salud debido a la discriminación por ser } \\
\text { trabajadora sexual }\end{array}$ & 12,9 & 14,5 & 27,9 & 20,0 \\
\hline $\begin{array}{l}\text { La derivaron a asistencia psicológica por } \\
\text { ser trabajadora sexual }\end{array}$ & 6,7 & 22,6 & 24,2 & 18,9 \\
\hline $\begin{array}{l}\text { Un profesional de salud informó a otras } \\
\text { personas que usted es trabajadora sexual }\end{array}$ & 8,3 & 17,3 & 19,5 & 15,8 \\
\hline $\begin{array}{l}\text { La derivaron a un servicio de infectología } \\
\text { o de enfermedades sexuales porque es } \\
\text { trabajadora sexual aunque la consulta } \\
\text { fuera por otro motivo }\end{array}$ & 6,7 & 11,3 & 21,6 & 14,6 \\
\hline $\begin{array}{l}\text { Le negaron la atención que necesitaba o } \\
\text { solicitaba, por ser trabajadora sexual }\end{array}$ & 6,3 & 6,5 & 21,1 & 12,8 \\
\hline $\begin{array}{l}\text { A1 contarle a algún médico que era } \\
\text { trabajadora sexual, la hayan tratado muy } \\
\text { bien }\end{array}$ & 54,6 & 40,3 & 43,0 & 45,4 \\
\hline
\end{tabular}

Fuente: Encuesta REDTRASEX, $2013\left(^{*}\right)$ Pregunta con posibilidad de respuesta múltiple. Los porcentajes no suman 100\%. (REDTRASEX 2013)

La experiencia más habitual es la de atenderse lejos de donde viven para evitar que en su casa o en su barrio se enteren que se dedican al trabajo sexual (mencionado por casi el $60 \%$ de las encuestadas). En sintonía con lo anterior, la experiencia mencionada en segundo lugar (44\%) fue la de optar por atenderse lejos de la zona donde trabaja para que en el servicio de salud no supieran a qué se dedica. Estas dos situaciones se vinculan con el secreto bajo el cual se intenta mantener la actividad, y evitar posibles situaciones de discriminación, ya sea por parte de las personas allegadas como por parte de los profesionales o personal de salud. En este sentido, el 33\% no quiso ir al hospital o servicio de salud para no tener que dar explicaciones sobre su actividad.

Ciertas experiencias de discriminación y hostilidad manifiestas fueron vividas por al menos un tercio: hostilidad por parte del personal administrativo, sufrir discriminación y tener que cambiar de hospital o servicio, y a un $13 \%$ les negaron directamente la atención. A veces no son los profesionales de salud quienes discriminan o dificultan el acceso, sino el contexto general obstaculizado por la persecución y estigma del trabajo sexual:

"Te lo digo porque a mí me pasó antes de hacerlo, que iba pasando por la clínica con unas amigas, las dos de pantalón corto, veníamos de la quinta costera, de un lugar turístico, y los policías.... nos hicieron montar al carro porque estábamos de pantalón corto y estábamos como sexys.

- ¿Yes porque querían aprovecharse...?

Claro 
Así lo hacen... agarran a las chicas, las recogen y les das tanto o sino vamos y estamos juntos.

- Eso es una violación...

Y lo tienen que hacer. En la patrulla. Yo digo que habría que concientizar o capacitar a los policías. O que haya algo, a un mayor al que tú puedas ir y decirle 'este hizo esto conmigo'.

- ¿El principal problema de salud es la policía...?

Sí, es la policía. Porque nosotras nos atendemos, y ellos no nos dejan estar en paz

Y también las que dan las citas. No mucho los doctores, sino las que dan las citas" (Panamá).

Hay experiencias más sutiles de discriminación: a un 15-20\% la derivaron a asistencia psicológica y/o de infectología sin otro motivo que el ser trabajadora sexual, o declaran haber sufrido violación de la confidencialidad, y a más de un tercio le recomendaron dejar el trabajo sexual. A veces tal "recomendación" es muy violenta, como muestra el siguiente relato de una trabajadora sexual que no se quedó callada ante la agresión:

“¡Tú ya no tienes edad de estar acá! ¿Qué haces que no estás en tu casa lavando platos? O preocupándome de mis nietos. Entonces yo agarré, cuando terminó, la quedé mirando seria y le dije, 'Usted hasta que pueda o hasta que muera', le dije. 'Porque yo, hasta que muera', le dije. 'Ahora, no sé usted', le dije. 'Pero no es forma de tratar, doctora'. (...) La doctora no tenía por qué tratarme así. Así tenga yo cien años, a ella no le interesa; me estoy haciendo controlar. Ahora, que yo siga trabajando, eso es mi problema ya. Eso no es problema de ella. Ahora si sigo cuidándome, es porque me amo, me quiero, ¿no?" (Perú).

La falta de confidencialidad también atenta contra la confianza en compartir en la consulta el tema del trabajo sexual:

“- Vos hace un rato decías 'no tengo por qué andar contando, es mi vida privada'. En los hospitales, si por alguna razón ustedes cuentan ¿se guarda esa información?

Un ejemplo, cuando iba a control y me tocaba hacerme una mamografía o una cosa de esas y si lo le decía que era trabajadora sexual, ella me anotaba un tic en la libreta, que yo no lo notaba pero que sí lo notaba el otro compañero. Era el código de ellas, como decir "la que te mando es una puta". Y te das cuenta por la forma en que te miran... te miran diferente

Te miran diferente... en el 2002 tuve una trombosis y se me ocurrió decirles, para que tuvieran una idea de quién era, de que era trabajadora sexual y me discriminaron. Mucho. Yo estaba en la cama y estaban ahí en junta médica y una doctora dijo 'cómo puede ser que sea prostituta...' y yo me sentí súper mal, me empecé a transpirar y me puse roja con todos los médicos ahí. Y otro doctor le dijo 'esto no tiene nada que ver, independientemente de lo que ella haga, en este caso estamos viendo por qué le vino la trombosis'.

- ¿Yen esos casos ustedes tienden a decirle "y a usted que le importa" o tienden a quedarse calladas?

En ese caso no dije nada porque estaba con mucho dolor en ese momento. Pero les dije que es mi forma de vida. Mi opción" (Chile).

Cabe destacar, finalmente, que el maltrato no es algo "inexorable". Casi la mitad de la muestra señaló que la han tratado muy bien cuando le contó a algún médico o médica que era trabajadora sexual.

Muchas de las mujeres, la mayoría, parecen no contar sobre su actividad laboral cuando van a atender su salud. Más de la mitad de las trabajadoras sexuales encuestadas dijeron que nunca habían sentido discriminación, rechazo o prejuicio por parte de médicos o personal de salud específicamente por ser trabajadoras sexuales. Por el contrario, el $31 \%$ ha experimentado este tipo de actitudes a veces y casi el $8 \%$ dice que siempre se sienten discriminadas o rechazadas en los servicios de salud. El porcentaje alto de quienes dijeron no haber sufrido discriminación envuelve dos situaciones: la alta proporción de quienes no dicen que son trabajadoras sexuales (porque no lo consideran relevante y/o para evitar discriminación o rechazo) como la de quienes, en menor proporción, han encontrado servicios y profesionales amigables, sean éstos específicos para trabajadoras sexuales o no. 


\begin{tabular}{|l|c|c|c|c|}
\hline $\begin{array}{l}\text { Distribución en valores porcentuales de las mujeres trabajadoras sexuales } \\
\text { encuestadas por frecuencia de experiencia de discriminación en servicios de salud } \\
\text { por ser trabajadora sexual, según subregión. América Latina y Caribe, 2013. }\end{array}$ \\
\hline $\begin{array}{l}\text { Subregión } \\
\text { Por ser trabajadora sexual, ha sentido } \\
\text { discriminación, rechazo o prejuicio } \\
\text { por parte de médicos o personal de } \\
\text { salud... }\end{array}$ & $\begin{array}{c}\text { Cono } \\
\text { Sur }\end{array}$ & Andina & $\begin{array}{c}\text { América } \\
\text { Central y } \\
\text { Caribe }\end{array}$ & $\begin{array}{c}\text { América } \\
\text { Latina y } \\
\text { el Caribe }\end{array}$ \\
\hline Nunca & 65,9 & 60,9 & 45,5 & 55,1 \\
\hline A veces & 23,1 & 28,4 & 38,4 & 31,6 \\
\hline Siempre & 2,3 & 7,7 & 10,3 & 7,5 \\
\hline No sabe, no desea contestar & 8,7 & 3,0 & 5,8 & 5,8 \\
\hline Total & 100,0 & 100,0 & 100,0 & 100,0 \\
\hline N & 264 & 271 & 464 & 999 \\
\hline
\end{tabular}

Fuente: Encuesta REDTRASEX, 2013

Dos de cada diez trabajadoras sexuales encuestadas afirmó sentir discriminación por algún otro motivo o pretexto no relacionado con ser trabajadora sexual, por parte de médicos o personal de salud. Dada la modalidad de la encuesta, tomada entre pares en condiciones de "semi-confidencialidad" (pues se conocen muchas de ellas al compartir espacios de trabajo y/o de activismo) puede presuponerse un subregistro de prácticas y condiciones de discriminación que prefieren mantenerse ocultas incluso ante la compañera encuestadora: por ejemplo en materia de $\mathrm{VIH}$, de uso de drogas u otras situaciones.

"En la medida en que no conocés tus derechos, vos considerás que es normal que te traten mal en un hospital o que te boludeen en cualquier lugar de atención al público. Segundo, vos pensás que te lo tenés merecido por ser puta. Hasta que después te das cuenta que no, que conocés que esto no es un delito, que tenés derecho empezando como ciudadana, y después como trabajadora. $Y$ te das cuenta de que todo lo que te venía pasando no es natural. $Y$ ahí es donde hacés un clic y te parás de otra manera, empezás a exigir cosas" (Argentina).

Entre quienes refirieron otras experiencias de discriminación, se destacan las migrantes.

\section{Discusión y conclusiones}

Los datos de la encuesta y los relatos recogidos muestran tanto la existencia de preocupación de las mujeres trabajadoras sexuales por su propia salud, como una actitud en principio favorable a acudir a los servicios de salud, en la medida en que no encuentren hostilidad en ellos y que éstos les brinden las respuestas esperadas.

De los datos del estudio no se desprende directamente en qué medida la atención es más o menos amigables en los servicios específicos para trabajadoras sexuales, que en los servicios no específicos, aunque en las entrevistas cualitativas surja una mayor amigabilidad en los países con cierto reconocimiento normativo (Uruguay) y una mayor hostilidad en contextos en principio más discriminadores (Panamá, República Dominicana). Algunos servicios son más amigables, en general en contextos de menor hostigamiento o mayor reconocimiento del trabajo sexual en la ciudad o país, y de mayor organización o empoderamiento individual y colectivo de las trabajadoras sexuales. Por otra parte, se señalan problemas en instituciones de salud con servicios u horarios específicos para trabajadoras sexuales, cuando éstas deben recurrir a atención o controles por fuera de los mismos.

Las situaciones enfrentadas, así como las estrategias para acceder a atención no hostil e incluso la considerada amigable y de buena calidad, son muy variadas, y sólo pueden analizarse dando cuenta de las 
trayectorias a lo largo del tiempo. El ocultamiento de la condición de trabajadora sexual es un recurso al que recurren las mujeres para evitar ser discriminadas o rechazadas, aun cuando esto pudiera tener consecuencias para la accesibilidad a la atención en salud. El estigma y la discriminación asociados al trabajo sexual llevan tanto a profesionales como a las mujeres a interactuar de manera tal que la calidad de la atención empeora y se vulneran así los derechos y la salud de las mujeres. Muchas veces se da la hostilidad manifiesta de instituciones y profesionales, y otras veces son las propias mujeres que se anticipan a tal hostilidad y prefieren no ir a atenderse, no consultar o no contar en la consulta que se dedican al trabajo sexual. También sucede que el temor al estigma o rechazo no refiera al sistema de salud, sino al entorno familiar, afectivo y de residencia (vecinas/os), por lo cual las mujeres suelen tener su actividad como trabajadoras sexuales y atender su salud lejos de su lugar de residencia, velando por mantener la discreción al respecto. Por ello también es cabe resaltar la importancia de la confidencialidad.

\section{Notas}

1. El equipo de investigación, coordinado por Mario Pecheny, estuvo integrado por Ximena Salazar Lostaunau, Albis Cruz, Inés Ibarlucía, Carolina Justo von Lurzer, Jorgelina Loza, Andrea Mariño, Elena Reynaga y las compañeras de la RedTraSex en los quince países donde se realizó el estudio. El equipo participó del diseño, pruebas piloto, capacitaciones, implementación y análisis, y los resultados se reflejan en un Informe final (REDTRASEX 2013). Las opiniones y juicios de valor del autor del artículo no necesariamente reflejan las posiciones institucionales de la Red, ni del Fondo Mundial contra el Sida, la Tuberculosis y la Malaria, principal financiador del proyecto. Agradezco a cada una de las compañeras la oportunidad de haber sido integrante de una muy feliz iniciativa de investigación participativa. Los resultados que aquí se presentan fueron compartidos con colegas de lberoamérica gracias al apoyo del Proyecto de investigación I+D Laboratorio Iberomaericano para el Estudio Sociohistórico de las Sexualidades, financiado por el Ministerio de Economía y Competitividad (FEM2011-27295).

\section{Bibliografía}

Barbosa, Regina (y Regina Facchini)

2006 Saúde das mulheres lésbicas. Promoção da equidade e da integralidade. San Pablo, Fundación Ford y UNFPA.

Bernstein, Elizabeth

2010 "Militarized Humanitarianism Meets Carceral Feminism: The Politics of Sex, Rights, and Freedom in Contemporary Antitrafficking Campaigns", Signs, nº 36 (1): 45-71.

Conill, Eleonor M.

2006. "Sistemas Comparados de Saúde", en W. S. Gaston y otros (orgs.), Tratado de Saúde Coletiva, Fiocruz/Hucitec: 531-563.

De Paiva, Laécia Lizianne (y otros)

2013 "A vivência das profissionais do sexo", Saúde em Debate (Rio de Janeiro), vol. 37, n 98: 467-476.

Morcillo, Santiago (y Carolina Justo von Lurzer)

2012 "Mujeres públicas y sexo clandestino: ambigüedades en la normativa legal sobre prostitución en la Argentina", en Daniel Jones y otros (eds.), La producción de la sexualidad : políticas y regulaciones sexuales en Argentina. Buenos Aires, Editorial Biblos: 89-121.

Pecheny, Mario

2003 "Identidades discretas", en Leonor Arfuch (coord.), Identidades, sujetos y subjetividades: narrativas de la diferencia. Buenos Aires, Prometeo: 115-142. 
Pecheny, Mario (y otros)

2010 Anticoncepción después de... Barreras a la accesibilidad a la anticoncepción de emergencia en la Argentina. Buenos Aires, Teseo-UNFPA-MSAL.

Petracci, Mónica (y otros)

2004 Calidad de atención en salud, Buenos Aires, CEDES/OPS, Serie Seminarios Salud y Política Pública.

Piscitelli, Adriana

2006 "Estigma e trabalho sexual: comentários a partir de leituras sobre turismo sexual", en Carlos F. Cáceres y otros (eds.), Sexualidad, estigma y derechos humanos: desafíos para el acceso a la salud en América Latina. Lima, FASPA/UPCH: 112-131.

\section{REDTRASEX}

2013 Estudio sobre estigma y discriminación en los servicios de salud a las mujeres trabajadoras sexuales en América Latina y el Caribe - Informe Regional 2013.

Varela, Cecilia Inés

2013 "Del tráfico de las mujeres al tráfico de las políticas. Apuntes para una historia del movimiento anti-trata en la Argentina (1998-2008)", PUBLICAR en Antropología y Ciencias Sociales (Buenos Aires), $\mathrm{n}^{\circ} 12:$ 3564. 